\title{
J
}

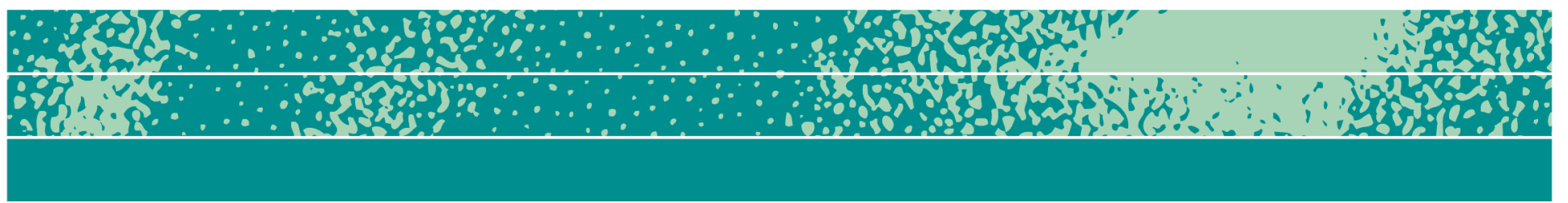

\section{Proceedings of the 2019 Global Business Anthropology Summit}

\author{
Timothy de Waal Malefyt and Robert J. Morais
}

\begin{abstract}
The second Global Business Anthropology Summit was held May 28-29, 2019 at Fordham University in New York City. The 2019 Summit brought together 160 industry practitioners and academic scholars to build upon the work of the 2018 Summit. The 2019 Summit was explicitly and emphatically forward thinking and action oriented to advance anthropological ideas in business. Its broad aims were to (1) advance thinking on the value of anthropological perspectives in business; (2) generate ways to spread appreciation of our work to academics, students, industry leaders, and the general public; and (3) build community among scholars and practitioners. The Summit's plenary panels and workshops demonstrated how anthropologists penetrate nearly every domain of business and are most adept at handling issues that are humanistic and complex. Throughout the two days, the Summit acknowledged the need to continue to grow the demand for anthropologists in business.
\end{abstract}

Page 1 of 34

JBA 8(2): 308-341

Autumn 2019

(C) The Author(s) 2019 ISSN 2245-4217

www.cbs.dk/jba 


\section{Introduction}

Applications of anthropology in, on, and for business are flourishing. Increasing numbers of anthropologists are employed by industry, and there are more anthropologists than ever in practice and academia who write about how businesses function (see Resources at www.businessanthro.com for books, journals, and videos). Business anthropology education, while still limited, has also expanded. Anthropology departments at US-based institutions such as the University of North Texas, Wayne State University, Clemson University, the University of Pennsylvania, and the University of Minnesota, and Duke University's sociology department offer courses on business anthropology. Business anthropology is also taught globally at Copenhagen Business School, University of Southern Denmark, BI Norwegian Business School, and HEC Montreal. US business schools at Notre Dame, Fordham, Columbia, and the University of South Carolina include anthropologists among their faculty. Ethnography, distinguished from anthropology, is part of the curriculum in business schools (Gebhardt 2015) and in design programs, as well as in other academic departments. This list of programs is representative, not comprehensive, and additional business anthropology training is likely to emerge. The numerous business anthropology sessions at recent meetings of the American Anthropology Association and the Society for Applied Anthropology and the growth of EPIC also attest to the increasing presence of anthropological ideas and methods in business, from practice, sustainability, ethics, and theoretical viewpoints.

In this context, Allen Batteau conceived and chaired the inaugural Global Business Anthropology Summit in 2018. His Summit report begins:

On April 24, 2018, 74 business anthropologists and students from nine countries met at Wayne State University in Detroit, Michigan to discuss the current state and future directions of business anthropology. This specialty-which has experienced substantial growth over the past ten years, with two new journals, an industry conference, and a major initiative at the annual meetings of the American Anthropological Association in 2017-is marked by both theoretical and practical challenges-and significant opportunities. (Report posted at www.businessanthro.com)

The 2018 Summit was foundational, covering a wide array of issues surrounding the application of anthropology in business. The Summit opened with plenary presentations on three overarching themes: (1) Practicalities of the work; (2) Market trends; and (3) Training the next generation of academics and practitioners. Workshops then explored each of the three themes. The closing plenary session outlined next steps based upon the workshops and looked ahead to the 2019 Summit. Batteau's report on the first Global Business Anthropology Summit is a 
valuable read for its description of what transpired and as a compendium of the major issues and opportunities for business anthropology in the $21^{\text {st }}$ Century.

The second Global Business Anthropology Summit was held May 28-29, 2019 at Fordham University, New York City, New York. With general business anthropology topics well covered in the first Summit, the 2019 Summit, co-chaired by Timothy de Waal Malefyt and Robert J. Morais, brought together an even larger group of industry practitioners and academic scholars to build upon the work done in Detroit and address more specific topics. The 2019 Summit was explicitly and emphatically forward thinking and action oriented. The broad aims of the event were to (1) advance thinking on the value of anthropological perspectives in business; (2) generate ways to spread appreciation of our work to academics, students, industry leaders, and the general public; and (3) build community among scholars and practitioners. The Steering Committee decided during the planning stage that the 2019 Summit should emphasize increasing demand for anthropologists in business and address issues such as training, standards, and ethics as they emerged organically or as panel and workshop leaders chose. Moreover, in designing the Summit, we aspired to not only generate fresh ideas but also to create roadmaps for anthropologists in business that could be followed after the Summit ended. Consistent with the 2018 Summit, rather than offer a conference with academic papers, the 2019 Summit included panels and workshops rather than paper presentations, reasoning that other forums, e.g., AAA and SfAA meetings, books, articles, etc., offer ample opportunities for scholarship.

\section{Agenda}

The 2019 Global Business Anthropology agenda (Appendix 1) featured a total of four panels and eleven workshops. In addition, during the plenary sessions, speakers included Edward Liebow, Executive Director of the American Anthropology Association (AAA), who voiced his support for business anthropology as an essential component of anthropology's vitality now and in the future, and Elizabeth Briody, Secretary of the AAA, who summarized a new initiative, Business Anthropology on the Road, which provides training in business anthropology to anthropology department faculty and job market preparation for anthropology students who want to apply their knowledge in business after graduation. (see Morais and Briody 2019).

\section{Sponsorship}

The 2019 Global Business Anthropology Summit would not have been possible without the support of its generous sponsors. We gratefully 
acknowledge the contributions of the following institutions and companies:

- Alfred P. Sloan Foundation

- American Anthropological Association

- Fordham University/Gabelli School of Business

- Wayne State University

- Kresnicka Research

- Menlo Innovations

- Weinman Schnee Morais

\section{Attendees}

There were 160 people in attendance over the two days of panels and workshops at the 2019 Summit, including those who pre-registered or "walked in" on one of the days of the Summit. That total was over twice the number who attended the 2018 Summit. We believe the robust participation in the 2019 Summit attests, in a large part, to the growing relevance of business anthropology method and theory in the business marketplace and to the practical nature of the Summit. Many of the Summit attendees were from the New York metropolitan area, but the New York City location enabled - and perhaps encouraged - people from other geographies to attend who otherwise might not have. As with the 2018 Summit, there was no registration fee, although unlike the 2018 Summit, attendees paid for their own travel. For the 2019 Summit, the list of invitees was far larger than the 2018 Summit; our objective was to make the 2019 Summit as inclusive as possible. Net, the 2019 Summit, even more than the 2018 Summit, brought together old acquaintances and generated many new connections. The scheduling of events allowed ample time for networking, which contributed to the overall sense of collegiality and future collaborative projects.

Virtually every domain of business anthropology was represented at the 2019 Summit: marketing, marketing research, advertising, business consulting, organizational culture, design, technology, user experience (UX), data science, risk management, finance, and more. Summit attendees included anthropologists working in corporations, consultants from companies large and small, and academics who write about but are less frequently, if ever, employed by industry. Also, ethical issues that anthropologists are adept at handling in business were addressed, such as how corporations can increase empathy with customers and employees; better training in diversity awareness; increasing acts of co-creation with customers; better elevating marginalized voices for ethical leadership, and humanizing technology, among other subjects. Many applied business anthropologists do not use "anthropologist" in their title or see it in their job description; that is evident from the titles included in the section below on panels and workshop participants. Notably, some Summit 
sessions discussed that explicitly identifying anthropology as a profession, rather than having it hidden or masked as some of the older generation felt they needed to do, would be beneficial to the discipline of business anthropology.

Among the Summit attendees were employees of and consultants for Facebook, Airbnb, Dropbox, IBM, Oracle, Verizon, ADP, WhatsApp, Instagram, WeWork, J. P. Morgan Chase, Hilton, and the Financial Times; representatives from the Boston Consulting Group, ReD Inc, Frog Design, Data \& Society, Kresnicka Research and Insights, Menlo Innovations, Cultural Keys, and Practica Group; and faculty and students from Fordham, Columbia, New York University, University of Pennsylvania, University of Minnesota, Wayne State University, University of North Texas, The Free University of Berlin, The Cooper Union, Johns Hopkins, the University of Washington, Universidad Iberoamericana, California State University, Davidson College, Monmouth University, CUNY, Universitat Autonoma De Barcelona, Clemson, University of Hong Kong, Central European University, Shujitsu University, University of Nebraska, Princeton, Yale, Cambridge, Temple University, and Illinois Institute of Technology. The full list of attendees shows an even wider range of companies and educational affiliations.

\section{Panels and Workshops: Aims and Outcomes}

The Summit plenary panels addressed how business anthropology might be defined, ways it solves business problems, its value in understanding technology, and the consequences of the work that anthropologists engaged in business do. The workshops explored a wider range of topics such as characterizing and marketing the value of the business anthropology, practice development and networking, user-experience, and diversity. Many of the panel and workshop topics were generated after the Summit Co-Chairs reviewed responses to a question on interests in the Summit registration form; additional topics were provided by panel and workshop leaders.

In advance of the Summit, panel moderators and workshop facilitators provided short statements of the going-in aims of their sessions. These statements were posted online in the Summit agenda so that registrants could choose in advance which workshops they wished to attend. After the Summit concluded, panel moderators and workshop facilitators crafted summaries of their session's outcome, i.e., what was discussed. The aims and outcomes are listed below, each authored by the panel moderators and workshop facilitators. Videos of some of the panels (those we were authorized to record) and PowerPoint presentations used in many of the workshops are posted at https://www.businessanthro.com/2019-summit.

Many Summit attendees intended to continue the panel and 
workshop discussions via email and other venues after the Summit ended. Additionally, face-to-face "meet ups" are being planned, beginning with a group in New York that will focus on specific topics in business anthropology.

\title{
Panels
}

\section{What is Business and Organizational Anthropology in the 21st Century?}

Moderator: Melissa Fisher, Visiting Scholar, The Institute for Public Knowledge, NYU; Consulting Anthropologist

Panelists:

Rachel Laryea, Doctoral Student, Joint Degree Program in Anthropology and African American Studies, Yale University

Grant McCracken, Consulting Anthropologist and Academic Without Portfolio

Gillian Tett, US Managing Editor of The Financial Times

Christina Wasson, Professor of Anthropology, University of North Texas

Caitlin Zaloom, Associate Professor of Social and Cultural Analysis, NYU

\begin{abstract}
Aims
The changing conditions of our world - the rise of the knowledge economy, the globalization and localization of business, as well as financial and environmental crises - are reshaping the meaning and role of business and organizational anthropology. This panel will explore different types of contemporary anthropological engagements with business and organizations, while simultaneously investigating their overlaps. It will address a number of questions: What exactly is business and organizational anthropology in the $21^{\text {st }}$ Century? What are the similarities and differences between an anthropology of business vs. the anthropology for business? What are the relationships between anthropologists and their interlocutors (e.g., clients) in the field of business, and how do these relationships shape the production of knowledge, writing, strategy making, and/or product design? And finally, how might business anthropologists forge partnerships in the future to build and extend business anthropology -- as an academic, "commercial" and/or ethically sustainable practice?
\end{abstract}

\section{Outcomes}

The workshop explored business and organizational anthropology in the 21st century. Drawing on their own career trajectories and experiences, 
each panelist discussed the intersections between anthropology and business. Gillian Tett explained that professions today train people to look at the world through tunnel vison. By contrast, cultural anthropology, she argued, is useful for looking at finance, technology and other businesses because it gives you "lateral vision." Caitlin Zaloom discussed her work on financial traders and new book on student debt in the United States. She argued that anthropological research means taking the perspective of the people who we work with, seeing the world from their specific location inside of it. This means that the insights anthropologists produce often times cut against the official description of how businesses or organizations work. Rachel Laryea spoke about the way her undergraduate experience and a year spent working at Goldman Sachs shaped her dissertation research on Black capitalists in the transAtlantic financial industry in the contemporary moment. Building on Zaloom's point, Laryea argued that cultural anthropology is an analysis of the discrepancy between how people narrate their lives and how they are organized institutionally - a definition easily applied to any study of business anthropology or the anthropology of business. Christina Wasson talked about her history of bridging different worlds, including linguistics and anthropology, academia and industry, ethnography and the design of technologies. Wasson addressed recent discussions about the differences between anthropology of business and anthropology for business, distinctions that, she maintains, are increasingly blurring. Grant McCracken argued that business anthropology needs to talk more about a model that is both academic and consulting. He explained that his own model has been to divide his life into two halves: consulting on the one side, and his own anthropology on the other. He noted that this model is changing. In the contemporary moment of an innovative economy and dynamic, disrupted culture, clients are increasingly interested to learn about what he does as an anthropologist.

\section{Anthropology at Work: Using our Toolkit to Tackle the Toughest Business Challenges}

Moderator: Derek Newberry, Adjunct Professor in Anthropology and Organizational Dynamics, University of Pennsylvania

Panelists:

Turi McKinley, Executive Director of Org Activation, Frog Design

Kaylie Wilson, Research Lead, WeWork

Martha Bird, Business Anthropologist, ADP

Tom Maschio, Owner, Maschio Consulting 
Aims

After decades spent in the trenches working with some of the world's largest corporations, the question: "What can anthropologists do for business?" is no longer a speculative one. Practitioners have helped Fortune 500 clients and employers better connect with their customers, engage employees, and rethink their strategies in increasingly complex market environments. However, most business anthropologists have been doing this work in isolation from each other, with no community of fellow-travelers with whom they can share insights, tools, and techniques, particularly outside of their own professional niche. This panel brings together experts from a wide range of sectors-management consulting, design, tech, and human capital-to build a common understanding about how we can apply our craft successfully in diverse business contexts. Our goals will be to: (1) share success stories and identify commonalities in how anthropologists are able to add value in the workplace; (2) identify ways to communicate this value effectively to clients and employers; and (3) share ideas on how to build effective networks of anthropologists across industry boundaries to continue building a knowledge base of practical tools and techniques.

\section{Outcomes}

In this panel, anthropologists and ethnographers from diverse industries explored what makes our toolkit effective in different organizational contexts and how we can better communicate this value to employers and colleagues alike. We noted first that while we represented a range of roles, from independent advisors to consultants in larger firms to inhouse researchers, we all had discovered the role of anthropology in our work serendipitously. Because our professionalization as anthropologists tends to be so haphazard, this reinforced the need to build communities of practice. We realized that what connected our diverse approaches was a common concern for putting people at the center of our work. We recognized that we often deal with clients and colleagues who view the customers and employees who are the focuses of our work more instrumentally. In these situations, we seek ways to help our corporate interlocutors build empathy through moments of connection with the customers and employees as holistic individuals. Finally, we acknowledged that this process works best when it is an act of cocreation-when we take clients along in the discovery process at key moments. What we should focus on next is discovering from a more practical standpoint how we can create more regular, easily accessible channels for sharing these ideas and practices.

\section{What Can Anthropology Offer Tech?}

Moderator: Yuliya Grinberg, Adjunct Assistant Professor, The Cooper 
Union

Panelists:

Jennie Doberne, Senior Design Researcher, Dropbox

Louise Beryl, Research Manager, Airbnb

Bryce Peak, Data Scientist \& Anthropologist in Hospitality, Hilton

Matt Artz, Director of UX, Cloudshadow Consulting; Founder \& Researcher, Azimuth Labs Susanne Cohen, Principal, UX Researcher, Elsevier

\section{Aims}

Industry and academia are increasingly recognizing the value of the social sciences in the technology sector. Companies like Twitter, Facebook, Spotify, Intel, and Microsoft to name just a few, are hiring anthropologists. At the same time, engineering schools and computer science departments across the country are integrating anthropology and social science coursework into their curriculum. This panel brings together anthropologists working in the tech sector and technology professionals with experience working with anthropologists to discuss the question: What can anthropology offer tech? Discussion will showcase the practical juncture of anthropology and technology. We will talk about initial stereotypes, unexpected surprises, internal challenges, and the impact of working as anthropologist and with anthropologists in the technological field. Conversation will also be speculative, discussing what anthropology could offer technology. Participants will engage in a "bucket list" challenge and share their wish-list for the role anthropology could play in the technology sector.

\section{Outcomes}

In a time when technology companies face growing public scrutiny, the idea that the age of machine learning needs the humanities is increasingly accepted as a foregone conclusion. This panel convened anthropologists working in the technology field to discuss what anthropology in particular can offer the tech sector. The panelists, who work as consultants, as data scientists, and as researchers, discussed how they applied their training to the professional space and dreamed together about the role anthropology may yet come to play in digital innovation. In the professional lives of the panelists, anthropological training often works its way into their work in unexpected ways, frequently exceeding the parameters of a particular research question as the place of work itself becomes an extension of their "fieldwork." Trained in making the familiar strange and the strange familiar, insight comes from the fresh perspective anthropologists bring, revealing, for instance, the many 
valances of a "page" for software developers or by pushing the concept of "dirty" and "clean" for a hotel brand. While the panelists all currently work in a research capacity, there was strong agreement that anthologists can and should rise to exceed design or UX research roles. The trained eye of an anthropologist alongside the field's inherent commitment to elevating marginalized voices are attributes valuable for ethical leadership. Still, none of the panelists currently identified as anthropologists in the workplace both because the title felt too narrow and too obscure. The question of how "anthropologist" as a professional identity may be elevated and consolidated remained open, ripe for future sessions of the Business Anthropology Summit.

\section{The Unintended Consequences and Social Implications of Company Actions: How Anthropologists Might Engage}

Moderator: Josh Kaplan, J.P. Morgan Chase

Panelists:

Patricia Ensworth, President, Harborlight Management Services

Abbas Jaffer, Facebook Research

Josh Kaplan, UX Research, JP Morgan Chase

Alexandra Mack, Senior Researcher, Ad Hoc LLC

Elizabeth Watkins, Researcher, Data and Society, and PhD candidate, Columbia University

Aims

A new museum comes under fire for displaying repatriated colonial objects; a technology giant pulls the plug on its plans for a second headquarters amidst vocal grassroots and anti-corporate advocacy; a FinTech startup is accused of mocking single people in a Valentine's Day ad. Many companies are under fire for the unanticipated consequences of decisions they've made, products they've built, or harmful outcomes they failed to prevent. The most notable examples-around data privacy and elections almost don't need to be mentioned. This panel will stimulate dialogue about how practicing anthropologists can help organizations anticipate and respond to the reverberating effects of the decisions they make and the products they build (particularly social, political, and cultural effects). Leaving critique aside, the focus will be on current and potential contributions practicing anthropologists might make. What differentiated value can practicing anthropologists bring to guide corporations in this type of terrain? How can that value be articulated so it is more likely to take hold among those who might employ anthropologists? What sort of teams might make the best foothold, in 
terms of receptivity of anthropology's contribution and the potential for maximizing impact?

\section{Outcomes}

Despite a skillset with the potential to make an important and distinctive business contribution, anthropologists are not consistently seen as a goto; a case in point is the February 19, 2019 call for help from Social Scientists that OpenAI published, listing eight disciplines, with no mention of anthropology. There are a several reasons for this lack of recognition-a topic that emerged throughout the Summit —including anthropology's self-siloing and distancing from science, and the stealth role that many anthropologists and anthropology itself has tended to play. Patricia Ensworth, described it: "In none of these engagements [e.g. in risk management] has my official job title ever included any reference to anthropology.....Yet my education and skillset as an anthropologist has always enabled me to ask the sort of questions that identify requirements and stakeholders other colleagues often miss." Panelist remarks on the value anthropology could play tended to focus on "holistic approaches" or the tendency to bring into the scope of conversation and analytic frame topics, perspectives, cultures, affected parties, stakeholders that not typically brought into view. Besides broadening the frame (or a debate, a research engagement, an understanding of impacts), anthropologists' ability to reframe or question the typical framing of a topic was seen as an important contribution. For instance, Abbas Jaffer described how, in contrast to political scientist colleagues, framing an election as an event rather than just a formal political exercise helped bring to the fore how it can disrupt everyday ways of interaction with communities or government; he credited this framing as "a big way we were both able to contribute to leadership making decisions." A thread throughout the panel was the future of work for current and aspiring practitioners. The emergence of safety and integrity teams in tech companies was cited as a clear opportunity, but it was observed that companies do not often invest in such functions, or in risk management, until they face a crisis. In the current climate of growing public awareness of the harm tech can cause or enable, practitioners can seize the moment to contribute from "where they already are", as Alexandra Mack put it, whether it be in UX, market research, outside consultancies, or independent research organizations.

\section{Workshops}

\section{Building on the Detroit Summit}

Facilitators: Allen Batteau, Department of Anthropology and Julia C. Gluesing, Department of Industrial and Systems Engineering, both at Wayne State University 
Aims

The 2018 Global Business Anthropology Summit in Detroit identified three major areas that our community could begin working on:

(1) Making our voices more important; (2) Advancing the training of Business Anthropology practitioners and scholars; and (3) Creating resources for promoting Business Anthropology. The Summit report identified three to six items for each of these, for a total of 13 potential action items. This workshop will promote a discussion of these three areas and the related action items from an orthogonal point of view: Advancing training, for example, while certainly of academic interest, would become yet more interesting if we can hear from industry voices as to how the training of scholars and practitioners should be advanced. Similarly, from an academic perspective, what resources would be most accessible for promoting Business Anthropology? In this workshop we propose to problematize Business Anthropology, by examining it from new (and potentially unexpected) viewpoints.

\section{Outcomes}

The Detroit Summit identified three areas where work could begin immediately:

- Making our voices more important

- Advancing the training of Business Anthropology practitioners and scholars

- Creating resources for promoting Business Anthropology

Some of the steps that have been taken in the past year and that could be taken in the future to advance these objectives include:

- Continuing the "Business Anthropology on the Road" initiative, launched by Robert Morais and Elizabeth Briody, making presentations at university anthropology departments to better familiarize them with this rapidly emerging subfield.

- Participating in the op-ed project being promoted by Daniel Ginsberg at AAA, an important initiative for business anthropologists to make our work known.

- Publishing case studies in Wired and Fast Company to reach newer audiences for Business Anthropology.

- Publishing book and article reviews in the Journal of Business Anthropology and on the business anthropology website to increase traffic and in journals of disciplines adjacent to anthropology to create awareness.

- Familiarizing anthropologists with current trends in Big Data and improving the quantitative abilities of anthropologists. Since anthropology is designated as a STEM discipline, there is 
opportunity for existing university programs to apply for funds to include courses in quantitative and mixed methods data analysis.

- Training managers in the value and values of anthropology through the Academy of Management and applied professional business organizations.

- Encouraging anthropologists to identify in their job titles and tag lines as business anthropologists.

- Increasing the visibility and usefulness of the Business Anthropology website as a common platform for collaboration and developing a shared identity.

- Promoting cross-disciplinary collaboration with designers, management scholars, engineers, and non-academics.

- Inviting participation from global voices and those from the margins to better contextualize our perspectives and our work.

In sum, there are many valuable efforts underway, and building and maintaining the network of Business Anthropologists is at the heart.

\section{Defining the Emerging Community of Business Anthropology}

Facilitators: Alexandra Mack, Ad Hoc and Ann Jordan, Emeritus Professor of Anthropology, University of North Texas

Aims

This workshop will focus on creating a definition around Business Anthropology, identifying emergent trends in our work and clarifying what needs of the community are not being met by other existing organizations. Building off this definition and needs, we will explore what format/structures will best address the perceived gaps moving forward. Is it a website, an online collaborative space, an annual conference, an organization with a leadership structure? What relationships should we have with other organizations? We will be aiming to articulate specific recommendations and next steps.

\section{Outcomes}

Our goal in this workshop was to define the business anthropology community well enough to articulate its unmet needs and the formats and structures that could address those needs. In the session, we began with the question "Why are you here?" as a lead in to that articulation. The group then generated a list of trends in Business Anthropology. From these trends a set of needs emerged, including the ability to bring together a global Community of Practice that has a focus that is both encompassing of practitioners and emphatically anthropological, and a desire for accessible resources including professional development, 
hiring, and spreading the word throughout the community. We then discussed what formats and offerings would be particularly relevant for people in business. Participants wanted to have ongoing discussions beyond annual meetings, and a resource center for jobs, training, publications, and networking. There are several existing formats that the community could better leverage, including the American Anthropological Association, the Journal of Business Anthropology, and the Business Anthropology website ( https://www.businessanthro.com).

The workshop ended with these specific recommendations and next steps:

Recommendation 1: Leverage existing resources more fully Next Steps:

- Ask for more on the website, in particular to build out the resources around jobs, training, publications, and networking

- Appoint a liaison to the AAA to work the association on creating more formats of interest outside of their annual meeting

- Encourage greater submission and readership of JBA

Recommendation 2: Look deeper into support structures for Communities of Practices

Next Step:

- Sue Squires has volunteered to research the literature around CoP support structures including Etienne Wegner's more recent work with digital communities

\section{Rebranding and Building a Marketing Strategy for Business Anthropology}

Facilitators: Melissa Vogel, Clemson University/Great Heron Insights and Adam Gamwell, Missing Link Studios

Aims

How do we "sell" anthropology? Anthropology desperately needs to rebrand itself. We need to clarify to the public that not only do we study human cultures at home and abroad, but we also have much to contribute to organizations struggling with hot-button issues like diversity and inclusion, corporate social responsibility, and user-centered design. We need to reclaim and "own" the contributions that originated in our discipline, like ethnography and the holistic approach. In this workshop we will identify the current obstacles to the spread of anthropology as 
essential to the business world and develop actionable strategies for breaking through those barriers. Participants will leave with tools and concrete steps they can take to open opportunities and move the discipline forward.

\section{Outcomes}

The workshop facilitators provided an overview of anthropology's branding problem and led participants through exercises on personal branding as well as systems level value propositions. We unpacked the historical shift between a more public anthropology of Margaret Mead and Franz Boas and how practitioners today can learn from their examples. To build on this, the facilitators crowdsourced some of the key concepts developed by anthropologists such as culture, emic and etic, and ethnography, and discussed how they could be reclaimed and branded as explicitly grounded in anthropology, to add value to business contexts. Participants then got hands on practice learning how to craft elevator pitches or personal branding statements in pairs and shared them for feedback. The workshop then unpacked systems level marketing strategy. Groups then designed value propositions targeted at one of the following five specific audiences: journalists, faculty, consultancies, industry decision makers and colleagues in other disciplines. The results of this exercise are available at businessanthro.com

The call to action for participants was to commit to regularly posting about their own work, or anthropology more broadly, in the venue and time interval of their choosing. Venues we discussed included LinkedIn and Facebook, blogs like Medium and Anthropology News, podcasts like This Anthro Life, and digital magazines like Sapiens and Scientific American. We have already noticed some participants fulfilling their commitment and plan to follow up with collaborative support to continue their marketing efforts and build greater cohesion in the business anthropology community.

\section{Communicating the Value of Anthropology Through Visual Thinking and Images}

Facilitators: Beth Holland, CultureWax and University of North Texas and Gigi Taylor, Luminosity Research and University of North Texas

Aims

Anthropology has an image problem. The first question for many potential clients is "What is anthropology?" Often anthropology is understood only by what is seen: ethnography. Our challenge is to communicate that anthropology is not just how we collect data, but how 
we analyze data. The secret sauce to an anthropological analysis comes from our core theories and concepts. One way to communicate the value of anthropology theory is through images, simple enough to be sketched on the back of a napkin. In this interactive workshop, we will first generate a list of potential concepts that are good candidates for visual representation. Then in small groups, we will sketch first drafts of theoretically inspired visual communication tools (diagrams, images, models, visual metaphors, etc.) The goal is to create simple, but powerful visuals for use in capability presentations, web sites, social media posts, or when all you have is a napkin.

\section{Outcomes}

The purpose of this workshop was to generate first drafts of visual representations of anthropology theories and concepts that could be used in capability presentations, web sites, and social media posts.

As a way to identify the best candidates for visual representation, participants were asked to list the theories or concepts from anthropology that have previously informed their research. The group generated an impressive collection of diverse theories. The most popular theories clustered around three areas: 1) Context: the social construction of reality, situated knowledge, implicit meaning, cultural categories, framing; 2) Symbolism: semiotics, symbolic communities, metaphors, Mary Douglas's symbolic pollution; and 3) Rituals of transformation: liminal time and space.

In preparation for the visualization exercise, the participants were given examples of visual thinking strategies (diagrams, abstract analogies, visual metaphors, etc.). Anthropology's classic iceberg metaphor of culture was offered as a good example of visual communication. Then anthropologists were briefed on the creative ideation process and encouraged to "concept" how anthropology could be visually represented. Drawing skills were not required.

Several strong ideas emerged from the concepting exercise. For example, Actor Network Theory was imagined as a pinball machine in constant motion. Turner's liminality and ritual process also generated various compelling visual representations. The next step will be to collaborate with a graphic artist to develop the strongest concepts into a collection of professional images that can be shared with the business anthropology community.

\section{Designing and Starting Your Practice}

Facilitators: Sabrina Nichelle Scott, Fullest Potential Consulting, Inc. and Matt Artz, Azimuth Labs 
Aims

Designing and Starting Your Practice is both an introductory and interactive workshop targeted at graduate students, early career anthropologists, and mid-career anthropologists who are interested in starting their own consultancy/agency. The objectives of this workshop are 1) why should you start a business, 2) types of mindsets that encourage business success, 3) ways for you to identify and prioritize what problems to solve, and 4) strategy creation and positioning.

You will participate in discussions and exercises to facilitate strategy formation and early steps of starting a business. The facilitators will share concrete examples to provide you with insights and tactical suggestions for developing your practice once it is up and running. At the end of this workshop, you will have an overview of the methods and tools that are useful for designing a business and starting it.

\section{Outcomes}

Early career and mid-career anthropologists attended this workshop. Some attendees did not have a business idea, while others have start-up businesses. There were a couple of attendees who wanted to share information with their students. Workshop topics covered reasons to start a business, skills and mindsets that contribute to business success, industries and emerging areas of practice, how to identify a problem to solve, and methods for designing and iterating on business models.

Participants engaged in group exercises that included identifying practice areas of interest, exploring reasons for starting a business, finding a viable business idea, and defining a value proposition. Although participants know there are many opportunities for consulting, and they have great ideas, some were stuck on what were the next steps. Based upon questions including monetization, hourly rates, and business development, the facilitators realized that there is a need for anthropologists to acquire more business, technology, and design skills in academic programs. There is also a need to establish internship partnerships with small consulting firms as well as larger corporations. Since there wasn't enough time in the workshop to adequately address measures in starting the practice, there are opportunities to offer separate workshops focusing on designing a practice and starting a practice. Participants were given additional information about national resources to help aspiring entrepreneurs and small business owners to develop their business ideas and start their consultancies.

\section{(Re)Defining the Value of Anthropology in Business}

Facilitators: Susan Kresnicka, President and Karis Eklund, Research 
Manager, both at Kresnicka Research \& Insights

Aims

When we frame anthropology's relevance to business as a single qualitative research method, ethnography, we limit the range of ways anthropology can create value. In this session, we will collectively brainstorm ways to articulate the value of anthropology in business overall, within specific business units, and across various industries. Participants will walk away with a fresh vocabulary for communicating the value of their services and perspectives.

\section{Outcomes}

In this workshop, participants processed and synthesized their understanding of the value of anthropology in business today. The workshop began with a general discussion that generated a wide range of ideas about current understandings/misunderstandings about the value of anthropology in business, why it can be difficult for us to articulate our value, and how we want the value of anthropology in business to be seen. The session concluded with break-out teams crafting pithy statements about the value of anthropology in business.

In the broad, stage-setting conversation, participants acknowledged an array of challenges in both our ability to articulate anthropology's value in business and our ability to convince the business world of it. First and foremost, lack of awareness surrounding what anthropology is and does makes anthropology seem irrelevant to, if not overtly out of place in, business. This suppresses practitioners' inclination to call themselves "anthropologists" in business settings, which in turn perpetuates the lack of awareness. When understood at all, anthropology is often perceived either as a "nice to have but not absolutely necessary" research technique (ethnography) or as relevant only to a handful of business domains (mainly research and organizational culture). Beyond external perceptions, the value of anthropology can be difficult to articulate because it arises, in part, from illuminating invisible dimensions of human social experience - we must convince people that we can see things they cannot. Moreover, we have to make the case that those otherwise invisible things we are uniquely qualified to see can impact the business's bottom line, a case that can be difficult to make given the distance between higher-level forms of value that people derive from the products, services, and experiences that businesses offer and the quantifiable, transactional events upon which business performance is measured. Finally, in this hybridized space, tension between anthropological and business norms (e.g., prioritizing 'complexity' vs. 'simplicity', critiquing capitalism vs. lionizing it, academic 
vs. business jargon) can undermine both our willingness to embrace and our ability to express our value.

Despite these challenges, some workshop participants possess a strong existing vocabulary for articulating their value in business, one that emphasizes anthropology's unique explanatory power (e.g., uncovering the 'why,' explaining behavior), focus on human beings, ability to reveal the 'invisible' (e.g., tacit assumptions/mental models, blindspots, lateral thinking), and capacity for bridging varying worldviews (between consumers and brands, employees and employers, varying business units, etc.). Whether they currently articulate the value of anthropology in business with fluency or struggle to even broach the conversation, participants were asked to draft (in break-out groups) a clear, onesentence value proposition statement for business anthropology. Taken as a whole, the resulting set of statements depict business anthropology as a unique - yet essential - way of thinking that helps businesses identify new opportunities, enhance cooperation, overcome obstacles, and mitigate risk in order to generate value for the organization and build stronger, more enduring bonds with the people they serve.

\section{What Business Anthropology Can Learn from Behavioral Economics (and Vice-Versa)}

Facilitators: Patricia Sunderland, Cultural Research \& Analysis, Inc. and Sris Chatterjee, Gabelli School of Business, Fordham University

Aims

Behavioral economics draws on the behavioral and social sciences to analyze how people make economic decisions. Popular books and articles have made the public aware of the appeal of its explanations. Academic programs in universities are expanding to include Behavioral Economics in their pedagogy. Industry has also embraced the emergent field with job creation and specialization. Behavioral economics could teach business anthropologists a thing or two about how to frame what we do for popular, business and academic audiences, which could help merchandize business anthropology and increase demand for our services. In this workshop, an expert on business economics will provide a brief overview of the field and we will then focus on what business anthropology can learn from the successes of behavioral economics and what we, as business anthropologists, can contribute to business economics. Audience participation in small exercises and discussions will enliven the session.

\section{Outcomes}

This workshop began with an overview of behavioral finance by Sris 
Chatterjee. He presented Hersh Shefrin's three themes of behavioral finance: Heuristic Driven Biases (people use rules of thumb), Frame Dependence (form is important), and Inefficient Markets (prices may not reflect value). Sris then explained ways these "beyond the simply rational" explanations for market activity have led to important recent developments in finance, including research on market sentiment and psychology. Sris recalled the earlier work of Benjamin Graham who coined the metaphor of "Mr. Market" and "his" mood swings in order to describe the euphoria and depression in market prices. Benjamin Graham's philosophy of value investing was made famous by legendary investor Warren Buffett. After these overview explanations a number of examples were provided including the case published in the Journal of Finance in 2001, "Massively Confused Investors Making Conspicuously Ignorant Choices." This title played on the MCI-MCIC ticker symbol confusion of Mass Mutual (MCI) and MCI Communications (MCIC). Investors were responding to information released by MCIC, but MCI moved in price after investors mistakenly confused the ticker symbols. Sris also talked about how new research in Economics and Finance is focusing on narratives. Robert Shiller, winner of the Nobel Prize in Economics has a paper titled "Narrative Economics" and a worldrenowned finance professor from NYU has recently published a book titled "Narrative and Numbers." This is a potentially useful area of research where economists and anthropologists can collaborate with one another.

After this overview, Patricia Sunderland provided a "cultural glimpse" of Behavioral Economics with a focus on two books, Freakonomics by Steven Levitt and Stephen Dubner and Misbehaving by Richard Thaler. She pointed to the cultural successes of these works, e.g., New York Times bestsellers, a popular National Public Radio show and a popular blog, the embrace by governments and policy makers, the establishment of a think tank and agency for international projects, and a Nobel prize in economics (a Nobel prize in economics was also won by Daniel Kahneman, author of Thinking, Fast and Slow.)

Prompted by some starter ideas of factors that made these works appealing to popular audiences in the US, workshop participants then participated in brainstorms of lessons that anthropologists could offer as well as learn from behavior economics. Lessons to offer included bringing in more diversity and multiplicity via culturally-based motivational views and the comparative method. Ways of going beyond the individual and really examining the social relations between parties in an exchange was also mentioned. Bringing in classic economic anthropology perspectives as well as more recent theorizations as seen in the sharing economy literature were also brought forward as very important to consider and as potentially helpful and enlarging perspectives for behavioral economics. In terms of lessons that could be learned, participants discussed the value 
of making the complex simple as well as devising frameworks that aided understanding and made matters concrete. Both the value of "distillation that comforts" of well as emotional shock value were suggested, as well as the potential of the phrase "cultural science." To the provocation that anthropology also needs a popular prize, it was suggested that what would truly be desirable was for an anthropologist, not only psychologists (among social scientists), to win a Nobel Prize in economics.

\section{The tl;dr (Too Long; Don't Read) of UX Research}

Facilitators: Jennie Doberne, Dropbox and Louise Beryl, Airbnb

Aims

What do anthropologists do in technology companies? This workshop will orient participants toward user experience and design research, a rapidly growing career path. We will explore questions such as: what is User Experience (UX) research? How does it differ from ethnographic research in academia? Is this role a good fit for your skill set and as a future career? Using real world examples, participants will work together to frame research questions and pair methods to questions in order to design a product research plan.

\section{Outcomes}

As anthropologists look beyond the academic job market, emergent fields such as user experience or UX research have become in-demand sites of employment. Making this transition requires translating and building upon the traditional skill set gained through anthropological training. This workshop articulated some of the similarities and differences between these pursuits, by drawing upon the presenters' situated experiences as UX researchers who have worked at multiple tech companies in the Bay Area. Anthropologists are well-equipped to enter fields within the tech sector. Yet this transition requires adjusting to a new cultural realm of practice that is both familiar and strange, as evoked by the widespread tech expression "tl;dr" ("too long, don't read").

In both realms. research follows a similar trajectory from research design to data generation, data analysis and reporting. Yet this workshop explored several key dimensions of how UX research (within tech companies) differs from most academic research. First, research in tech is often team-oriented and highly collaborative in nature, as UX researchers are embedded within product teams, consisting of multiple roles (e.g., developers). Second, the speed of work - demanding results in short time frames-is unlike most academic timeframes. Finally, tech research produces actionable insights and recommendations that a multi- 
disciplinary team can understand and act upon in order to build product. These findings, or deliverables, are consumed by the working team and stakeholders more broadly. While a concise, written form is common, additional creative and interactive formats enable teams to gain understanding and make the findings tangible and memorable.

\section{A Practical Toolkit for Discovering Blindspots and Driving Innovation}

Facilitators: Inga Treitler, Anthropology Imagination LLC and The University of Tennessee,

Elizabeth Briody, Cultural Keys LLC, and Jo Aiken, University College London

Aims

Assumptions about what people call normal, create blindspots that inhibit clear communication, product innovation, adaptation within organizations, and more. In small breakout groups you will dive straight into a hands-on discovery of blindspots from two industry cases. These hands-on exercises will allow you to build a practical toolkit for turning a critical lens on yourself as researcher. The toolkit will serve as a module for training and equipping clients and partners to identify and illuminate their own blindspots. Disrupting blindspots is advantageous for organizations and enterprises because it signals the importance of adapting to changing practices and products. This toolkit's practical approach contributes to anthropology's value in the business world.

\section{Outcomes}

A blindspot exists when something is so familiar and seemingly so logical that you consider it an unassailable truth. In this workshop, we focused on blindspots because they are easy to relate to personal experience and may be helpful in conversation with clients. The workshop was organized into two 45-minute segments.

We began by exposing our own and others' assumptions implicitly, blindspots - by listing "10 things we know to be true." Eventually each participant introduced a neighbor, telling one thing the neighbor "knows to be true." A core finding was that the concrete, physical features of the world are easily accepted, but personal perspectives open dialogue and may yield change. In the second segment, we formed small groups to address a problem affecting us all in anthropology: the continued resistance within the academy to collaboration with businesses and organizations. The aim was to expose blindspots about the practice of anthropology. A core recommendation is that continued exposure to blindspots can be reduced through 
collaborative ventures, not unlike the discussions of " 10 things you know to be true." The next step, taken the day after the workshop, was to include findings about practice blindspots as part of a broader presentation to the AAA Executive Board. Several recommendations were made for increasing collaborations linking academia and practice, including joint field projects in organizational and business settings and co-authorship on reports and publications. Such collaborations may open the way to changes in course content and to the academy's reward structure.

\section{Networking in The Industry: Combining Anthropological and Coaching Tools to Create Strong, Powerful Connections}

Facilitators: Melissa Fisher, The Institute for Public Knowledge, NYU;

Consulting Anthropologist and Jim Arnoff, President, The Arnoff Company

Aims

This interactive workshop, co-presented by a business anthropologist and professional coach, will provide key insights to network and pitch with conviction for powerful results. Participants will learn how to apply anthropological tools to create a command of the networks in their industry; how to make the strongest connection quickly and how to pitch with conviction to make the most memorable interactions.

\section{Outcomes}

The purpose of this workshop was to explore the purpose and value of networking in professional settings. Melissa Fisher presented an overview of anthropological and sociological perspectives on networks and networking. She focused on two ethnographic case studies. Drawing on several decades of fieldwork with Wall Street women, Fisher discussed the strength of all female professional networks and the ways such networks provide women not only the resources necessary to learn to maneuver on Wall Street, but also a female space for the women to meet one another and build important ties and friendships with one another (Fisher 2012: 12). She also spoke about the work of anthropologist Christina Garsten who has shown, that in the world of think tanks, social connections in professional and powerful networks constitute an "economy of connections" where referrals, references and ties "take the form of valuable symbolic capital in a highly competitive and politicized form of exchange" (Garsten 2013: 151).

Jim Arnoff then proceeded to lead the group in an interactive portion of the workshop. He focused on helping participants to learn key tips for networking as well as communicating in professional interactions. 
He coached individuals on the kind of language to use (and not use). He also talked about how to let go of what might be standing in the way of communicating what individuals want in their academic and/or business careers. The workshop participants raised several pertinent issues regarding networking. We talked about the challenges anthropologists face in distilling complex ideas into relatively simple ones. We also spoke about learning to be more confident in presenting our ideas. We ended with a discussion of how structures of power shape and constrain networking practices in and out of academia.

\section{Diversity Doublethink: Policy Myths and Performance Rituals}

Facilitators: Melissa Fisher, The Institute for Public Knowledge, NYU; Consulting Anthropologist and Patricia Ensworth, Harborlight Management Services and Adjunct Instructor, NYU School of Professional Studies

Aims

This workshop will explore how anthropology can help turn hopeful thinking about diversity into realistic strategies. We will begin with a discussion of the ideals of diversity espoused in corporate mission statements, then contrast the actual processes by which cohesive teams evolve (and dysfunctional teams fall apart). We will then provide some exercises in which participants reflect upon the components of their identities and recognize the traits necessary for different types of effective teams and for career success in different types of organizations.

\section{Outcomes}

The purpose of this workshop was to explore the gap between ideals and realities in organizational behavior with regard to the concept of diversity. Melissa Fisher presented several case studies based upon her experiences as an academic expert whose services have been engaged by various types of clients seeking to increase the number of women in their ranks, provide products and services to women and/or portray gender and power in organizations. Specifically, she discussed providing: 1) branding expertise for re-imagining the hotel experience for female business travelers; 2) research on women in finance for Equity, a feature film about women on Wall Street; and 3) policy recommendations related to women and labor for the G20. Patricia Ensworth presented observations from her career as a project and program manager, management consultant, and leader of management training workshops, describing the different types of diversity initiatives which have proven 
effective in different business contexts. Both speakers demonstrated exercises managers can use to promote a climate of inclusion and improve communication among stakeholders across the organization.

Following the presentations, the workshop participants raised several pertinent issues for discussion. We considered the challenges faced by an external consultant in balancing organizational goals and personal ideological advocacy. We debated whether or not it was possible for a workplace to ever feel like a "safe space" for disempowered or marginalized employees even when their advancement to positions of greater authority is supported by organizational diversity programs. We analyzed the relevance of the classic Stages of Team Development model (Tuckman 1965) to ongoing incremental progress toward inclusion. We reflected upon the changes which occur in a global organization's shared understanding of the notion of diversity when the majority of leaders are not Euro-American. We questioned the potential impact of artificial intelligence, machine learning, and algorithmic decision-making on the achievement of diversity objectives within an organization. Many of these topics merit further exploration at future Global Business Anthropology Summits.

\section{Post-Summit Debrief}

Immediately following the close of the program, approximately 12 attendees convened to discuss what transpired in the Summit. Below are notes from that debrief:

1. The 2-day format worked well, balancing plenary sessions with workshops. The pace was leisurely which allowed ample time for networking.

2. Many agreed that the format was inclusive of attendees, e.g., a large array of participants was involved.

3. Perhaps the current Summit was more "inward-facing" and should work towards becoming more "outward-facing" for future Summits.

4. A point was raised to incorporate more of a social media presence during the conference, such as establishing a hashtag for spreading pictures, news, and activities.

5. The group discussed hiring a public relations firm to promote business anthropology, if funding can be generated.

6. The next Summit might target human resource managers and invite them to promote hiring.

7. The group discussed attracting future sponsors by offering that they bring us a specific business problem to solve. In return, they would receive analysis and recommendations from selected business anthropologists in attendance. 
8. Some among the group suggested identifying KPI's (key performance indicators) for measuring future Summit success (number of people in attendance; funds raised; number of subsequent publications; where publications appear; increased social media conversations).

9. It was suggested that one or more scholars address the academic issue of (lack of) employment for anthropologists in a highly respected academic publication, written by and from the perspective of a business anthropologist.

10. The group discussed a possible need to formalize Business Anthropology as a legitimate community (within or acknowledged by the AAA) with an elected board and its own set of statutes, guidelines, etc. No consensus was reached on this issue.

\section{Post-Summit Survey}

Anecdotal reports during and after the two-day event indicated that the "buzz" surrounding the Summit was extremely positive. Within a few days after the Summit ended, an online survey was sent to all of the attendees; approximately $40 \%$ of them responded.

Ratings of the Summit's overall quality, value to the respondent's career, and the experience of the 2019 Summit as compared to other conferences the respondent attended were mainly in the excellent and above average ranges. The panels and workshops fared well overall. Representative responses to an open-ended question (What was the biggest take-away for you from the entire Summit experience?) are shown in Table 1. 
Table 1. Representative Comments on "Biggest Summit Take-Away"

That Business Anthropology seems to have a brand identity issue---I hadn't really understood this so was glad for the warning and discussion.

Get to know the different possibilities business anthropology gives to a professional career

A large demand for convenings of this sort from a talented and eclectic array of practice areas

Community building with some clear action items

Opportunities to communicate the value of Anthropology.

What business anthropologists are doing is valuable to the economy and to society

How business anthropologists might work together across organizations

the field needs to be branded

I feel like we're making forward progress with business anthropology as a viable career that's just as valued

Most attendees I talked to reflected my take-away that this was a potential "home" for Biz Anthro

networking

The increasing and diverse number of jobs and interests that business anthropologists have.

How we must use concepts originating in anthropology (e.g., context, holism) to explain what we do to the

I learned how prominent anthropology is in other sectors and how to communicate its value to other

professionals not familiar with the field

There's a growing practitioner group that is advocating for value of anthropology in corporations. We need to professionalize and share tools, methods, and approaches!

Feeling permission to brand myself as a 'business anthropologist' even if it's not my title

It was immensely buoying to be in a room of professionals and academics who shared my intellectual

background and were doing smart, interesting work outside of academia.

There is a lot of potential for improvement within our field. Good inspiration for working more on spreading the word.

I am not alone. I have found my background in anthropology very useful in designing programs and running businesses but always hid my college major. The business environment may be ready for me to take off the how to better present what anthropology can offer business and industry

Business anthropologists must unite

Business anthropology is maturing as a field

There are some amazing people in this field and these meetings give us social cohesion \& common identity, keep us on the same page and keep the field moving forward

Anthropology needs to learn how to better communicate its value in industry beyond ethnography connecting people in business anthropology globally

the value of anthropological tool kits in diverse employment industries, tech, finance etc...

Building a common vocabulary is crucial for business anthropology to define itself as a field and community of practice; we need to build opportunities for one another, especially those seeking to work as consultants, The coalescing (finally) of a critical mass of business anthropologists to effect change.

It was great to see how and where other anthropologists are applying the tools of the trade within

Demand and opportunities for anthropologists in the private and non-profit sector are expanding continue to grow and we need to continue evolving the way we communicate our value

We need to come together more often, we're thinking about many of the same issues, and we need to collaborate in person and online more 


\section{Looking Ahead to Summit 2020}

The next Global Business Anthropology Summit will convene in 2020 at the Free University, Berlin, Germany. Co-chairs Mustafa Abdalla and Inga Treitler are formulating the agenda as of this writing, building on the learning of the first two summits, and expanding global horizons. During the closing plenary session of the 2019 Summit, Abdalla and Treitler presented an overview of the Berlin setting and encouraged all who attended the 2019 Summit to attend the 2020 Summit.

\section{References}

Batteau, Allen 2019. Report on the 2018 Global Business Anthropology Summit. Posted at www.businessanthro.com.

Fisher, Melissa. 2012. Wall Street Women. Durham: Duke University Press Garsten, Christina. 2013. All About Ties. Think Tanks and the Economy of Connections. In Organisational Anthropology: Doing Anthropology In and Among Complex Organisations. C. Garsten and A. Nyqvist, Eds. London: Pluto Press

Gebhardt, Gary. 2015. Yes, Virginia, We "Do Ethnography” in Business Schools. EPIC Perspectives. https://www.epicpeople.org/yes-virginiawe-do-ethnography-in-business-schools/

Morais, Robert J. and Elizabeth K. Briody. 2019. Business Anthropology on the Road. Anthropology News website, May 28, 2019. DOI:

10.1111/AN.1175

Tuckman, Bruce W. 1965. Developmental Sequence in Small Groups. Psychological Bulletin. 63: 384-399.

Timothy de Waal Malefyt is Clinical Associate Professor of Marketing at Fordham University, Gabelli school of Business, in NYC. He can be reached at tmalefyt@fordham.edu.

Robert J. Morais is Principal Emeritus of Weinman Schnee Morais and an adjunct professor at Columbia Business School. He can be reached at rmorais67@gmail.com. 
Appendix 1. Agenda

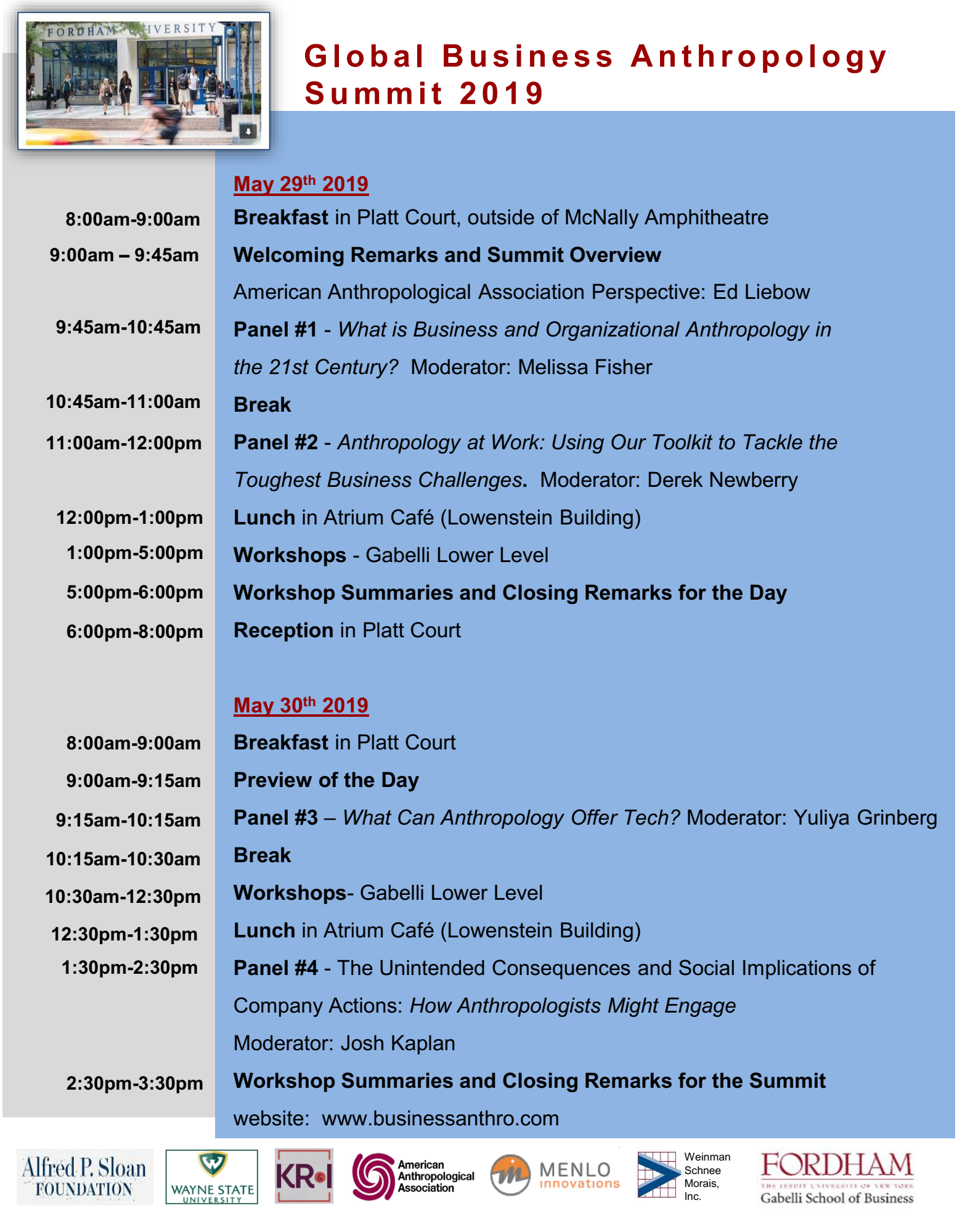




\title{
Appendix 2. Sketchnotes on the 2019 Global Business Anthropology Summit
}

\author{
Kendra Allenby
}

These sketchnotes were drawn in real time as the panels took place. Their goal is to take these verbal discussions and give them a visual record, one that hopefully captures important moments from the panels and makes these moments memorable, digestible, and shareable.

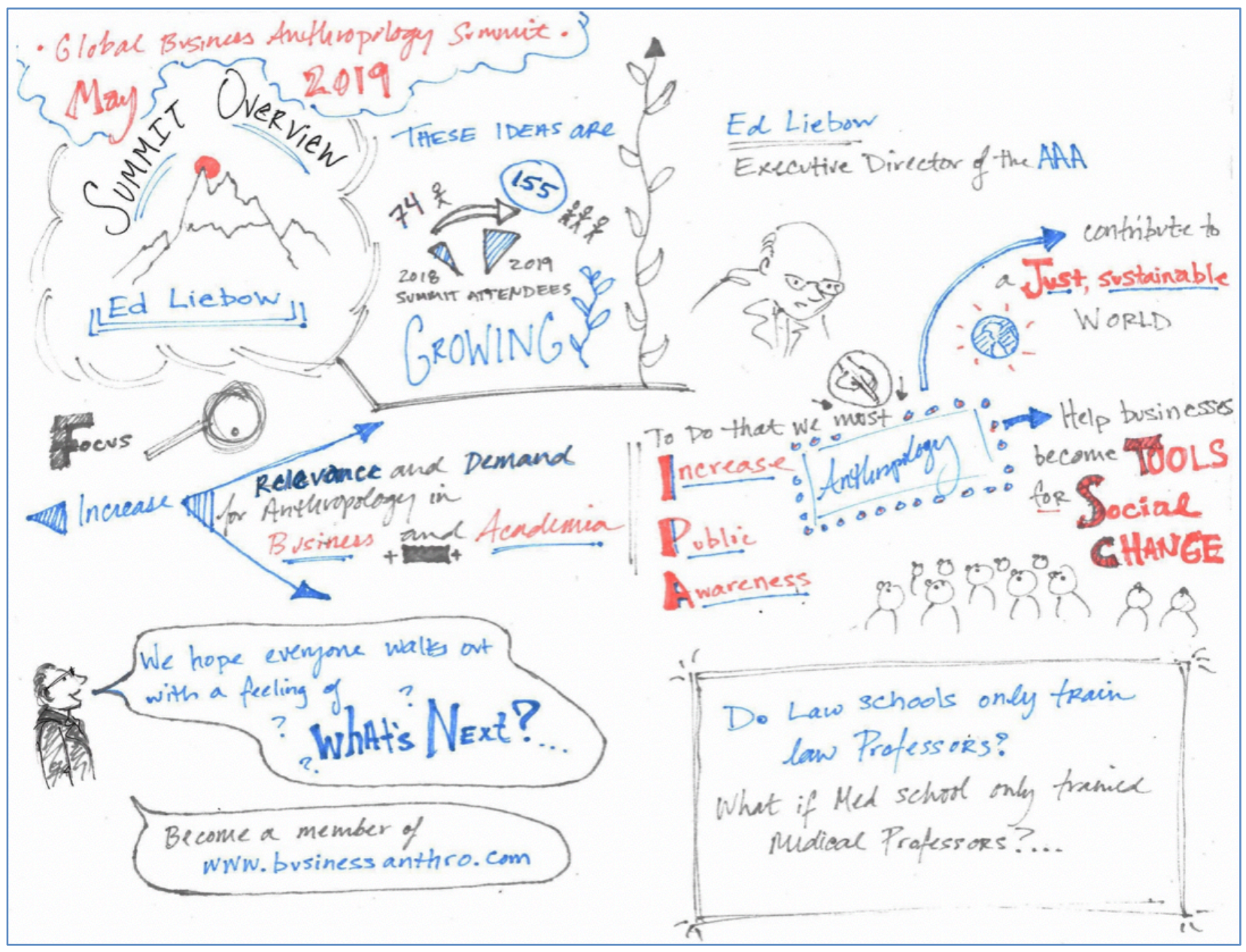



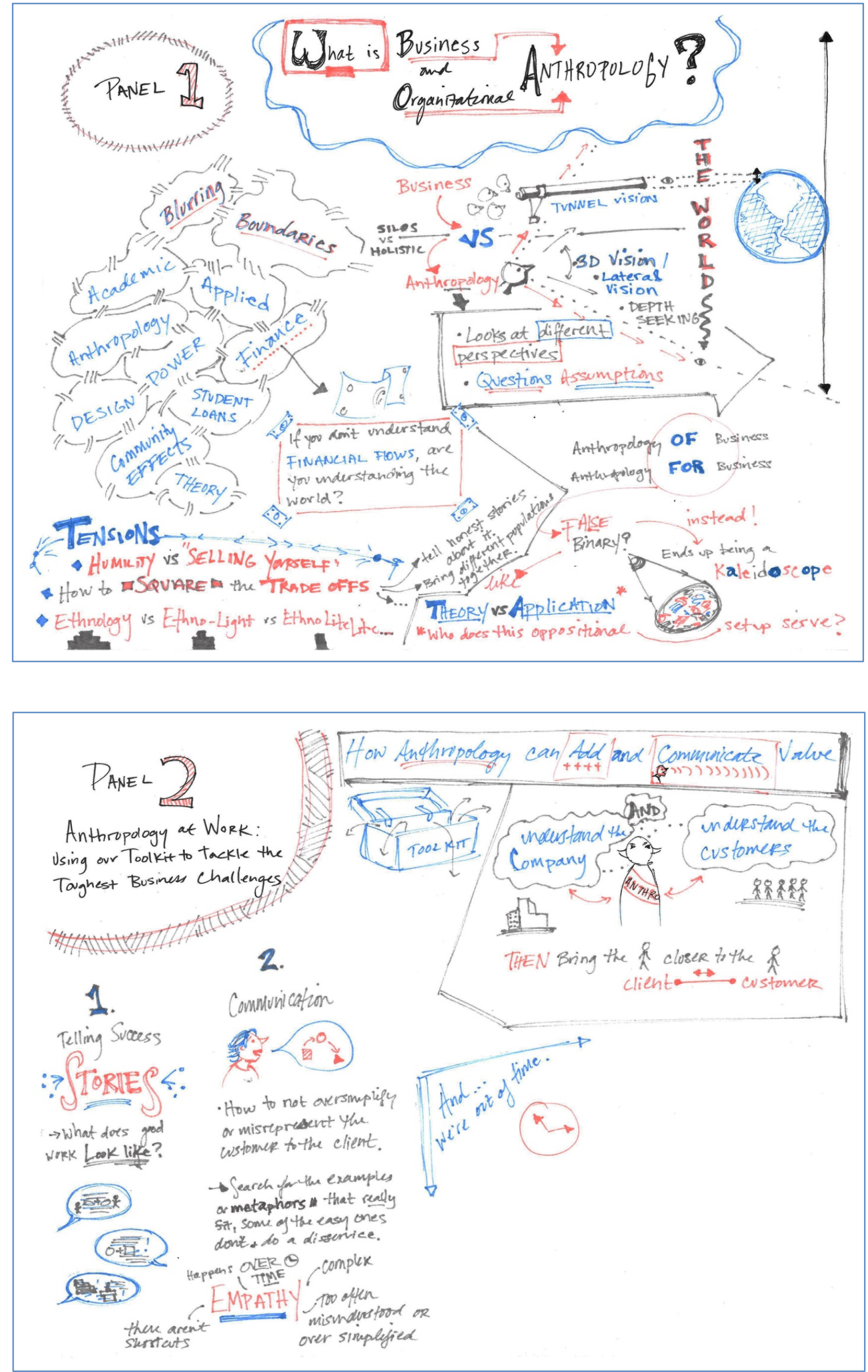

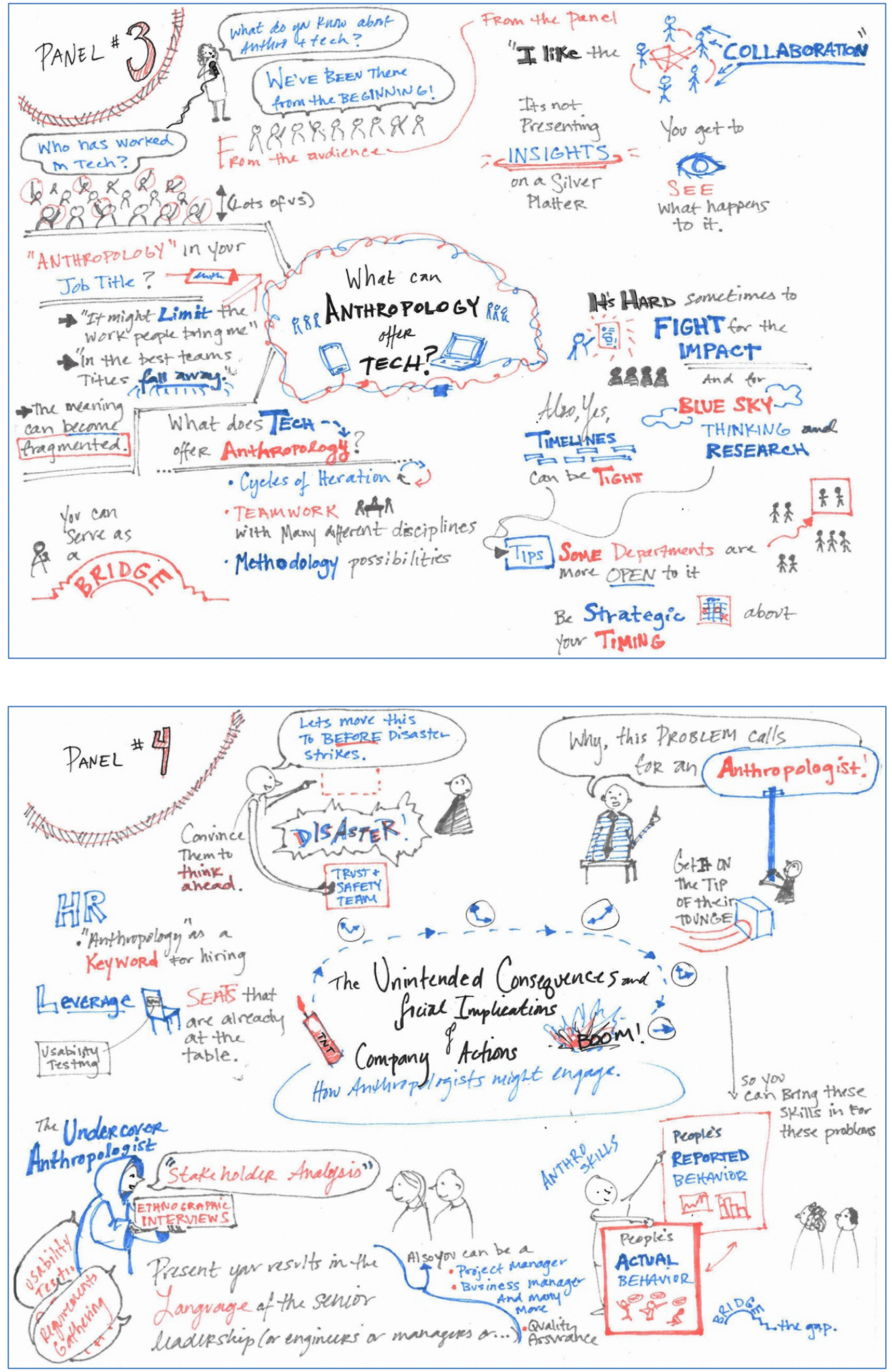


\section{Appendix 3. Acknowledgements}

In addition to the 2019 Summit sponsors listed above, we thank the following:

\section{Steering Committee}

The 2019 Global Business Anthropology Co-Chairs acknowledge with appreciation the contributions by members of the Steering Committee:
Allen Batteau
Elizabeth Briody
Patricia Ensworth
Melissa Fisher
Yuliya Grinberg
Josh Kaplan
Alexandra Mack
Derek Newberry
Barbara Olsen
Inga Treitler

\section{Panel and Workshop Leaders and Participants}

We thank the panel moderators and workshop facilitators who conceived and managed their sessions and wrote the statements included in this report. We also express our gratitude to the participants in the panels and workshops for their contributions.

\section{Additional Acknowledgements}

Recognition must be given and special gratitude expressed to Donna Rapaccioli, Dean of the Gabelli School of Business at Fordham, for graciously hosting the event; Sertan Kabadayi, Marketing Professor and Marketing Area Chair, whose openness to business anthropology encouraged this Summit to be held at Gabelli; and to Aida Lahood, Marketing Area Program Manager, Fordham University Gabelli School of Business, whose organizational expertise and dedication contributed immeasurably to the 2019 Summit.

In addition, we acknowledge the help of the following individuals at Gabelli: Dushinka Malhotra, who formatted the post-Summit survey, Akash Jethwani, who maintained the Summit registration list, and the 
people who contributed to the smooth running of the Summit on-site: Jay Tibbits, Cynthia Yue Qiu, Linda Agnew, Jennifer McKeown, Stephen McGuiness, and Maria St. Hilaire.

We appreciate the contribution to this report of cartoonist Kendra Allenby. For more on her work see: www.thedrawnoutdays.com and Instagram at kendra_allenby.

We thank Adam Gamwell and Matt Artz for their technical assistance with all things on www.businessanthro.com from design to posting to troubleshooting.

We extend our gratitude to Gillian Tett who opened her home to New York area anthropologists in business to connect and enjoy sumptuous meals. 